\title{
GRAVITATIONAL LENSES AMONG HIGHLY LUMINOUS QUASARS: LARGE OPTICAL SURVEYS
}

\author{
J.-F. CLAESKENS \\ European Southern Observatory (Chile), and \\ Aspirant au FNRS (Belgium), \\ Institut d'Astrophysique, Université de Liège, \\ 5 Avenue de Cointe, B-4000 Liège, Belgium
}

A.O. JAUNSEN

Nordic Optical Telescope, Ap. 474, S/C de La Palma, E-38700 Canarias, Spain

\begin{abstract}
AND
J. SURDEJ

STScI, 3700 San Martin Drive, Baltimore, MD 21218, USA, Member of the Astrophysics Division, Space Science Department of the European Space Agency, and Directeur de Recherche au FNRS, Belgium
\end{abstract}

\begin{abstract}
The search for multiply imaged quasars among highly luminous quasars (HLQs) is a very good strategy to determine the fundamental parameters of the Universe. We report on the present observational status of a combined sample of HLQs, including new observations obtained with the Nordic Optical Telescope (NOT) and at ESO. This combined sample of HLQs now contains 1178 distinct HLQs. A complete list of the total sample will be soon made available, through a World-Wide-Web page. Preliminary maximum likelihood results are also presented, using a simple statistical model to constrain the values of galactic parameters, of the number counts of QSOs, and of the cosmological constant.
\end{abstract}




\section{Introduction}

The systematic search for multiply imaged quasars among HLQs $\left(M_{V} \leq\right.$ -27 for $\left.H_{0}=50 \mathrm{~km} \mathrm{~s}^{-1} \mathrm{Mpc}^{-1}, q_{o}=0.5\right)$ has been in progress for 9 years, since its initial start in 1986 (Surdej et al. 1987). This technique has proven to be very efficient in order to find new gravitational lenses (GLs) (Turner et al. 1984, Surdej et al. 1987, 1988, 1993a). Today, the large number of observed HLQs also allows us to perform meaningful maximum likelihood analyses to constrain the parameter values of the lens and of the cosmological models.

In Section 2, we present the properties of the present merged sample of HLQs, together with those of the sub-samples. We then give in Section 3 a short overview of the main relations used to estimate the probability for lensing. Finally, we present, in Section 4 preliminary constraints on some galactic and cosmological parameters of the model, using the maximum likelihood analysis and give a short conclusion in Section 5.

\section{Survey sample}

Previous observations of HLQs have been conducted at ESO (Surdej et al. 1993a), with HST (Maoz et al. 1993), the CFHT (Crampton et al. 1992, Yee et al. 1993), and with the NOT (Jaunsen et al. 1995). The statistics of the merged sample has been improved by the observation of new HLQs, carried out with the NOT and at ESO, and by a careful re-analysis of the HST frames (see Surdej et al. in these proceedings).

The new observations have been incorporated in the respective subsamples presented in Table 1. The present merged sample contains 1528 observations of 1178 different HLQs. The last column of Table 1 shows the number (and the fraction) of HLQs kept in each subsample, after selecting the best observation among multiple ones of the same HLQ. It can be seen from this table that the average brightness of the observed samples is not declining from a lack of bright HLQs.

There are 6 lenses in the merged sample: UM673 (Surdej et al. 1987), PG1115+080 (Weymann et al. 1980), Q1009-025 (Surdej et al. 1993b), HE1104-1805 (Wisotski et al. 1993), Q1208+1011 (Magain et al. 1992) and H1413+117 (Magain et al. 1988). Also a few new promising GL candidates are being found, like J03.13 (Claeskens et al. 1995). The observed properties of these lenses can be found in Surdej et al. (1995), and also by connecting to the GL World-Wide-Web page at STScI (the URL address is given below).

The good correlation between intrinsically bright QSOs and high lensing probability is illustrated in Figure 1, which represents the probability for 
GRAVITATIONAL LENSES AMONG HIGHLY LUMINOUS QUASARS 15

TABLE 1. Individual properties of the sub-samples

\begin{tabular}{lcccccc}
\hline Sub-sample & $\langle z\rangle$ & $\langle V\rangle$ & $\left\langle M_{V}\right\rangle$ & $\begin{array}{c}\langle\text { FWHM }\rangle \\
\text { [arc-sec. })\end{array}$ & $N_{q}$ & $N_{\text {q,best }}(\%)$ \\
\hline $\begin{array}{l}\text { HST } \\
\text { (Maoz et al.) }\end{array}$ & 2.1 & 17.6 & -27.7 & - & 495 & $408(82 \%)$ \\
$\begin{array}{l}\text { ESO } \\
\text { (Surdej et al.) }\end{array}$ & 2.2 & 17.6 & -27.8 & 1.00 & 396 & $279(70 \%)$ \\
$\begin{array}{l}\text { CFHT } \\
\text { (Crampton et al.) }\end{array}$ & 2.4 & 18.1 & -27.4 & 0.66 & 101 & $81(80 \%)$ \\
$\begin{array}{l}\text { CFHT } \\
\text { (Yee et al.) }\end{array}$ & 2.2 & 17.7 & -27.8 & 0.76 & 104 & $32(31 \%)$ \\
$\begin{array}{l}\text { NOT } \\
\text { (Jaunsen et al.) }\end{array}$ & 2.0 & 17.5 & -27.6 & 0.90 & 432 & $378(87 \%)$ \\
\hline TOTAL & 2.2 & 17.7 & -27.6 & 0.90 & 1528 & 1178 \\
\hline
\end{tabular}

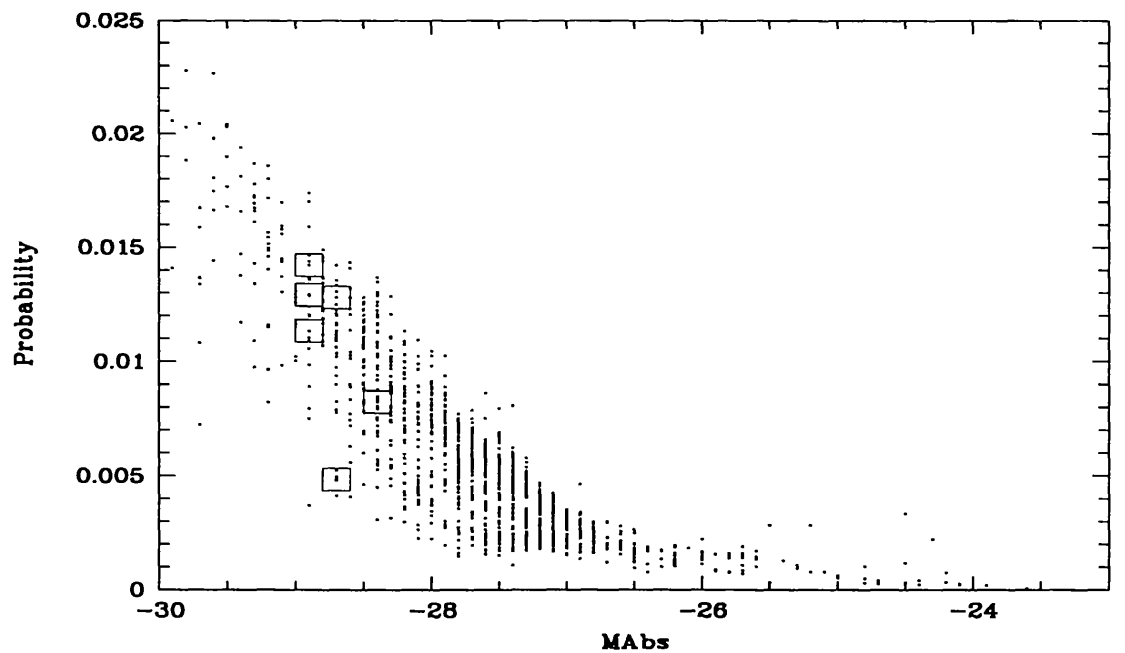

Figure 1. Plot of the absolute V magnitude versus the lensing probability for the 1178 HLQs. The 6 accepted GL candidates in the sample are marked by the squares

each HLQ to be lensed, versus their absolute magnitude. The 6 lensed QSOs in the sample are marked with a square.

A complete list of the total sample will be soon made available and updated through a World-Wide-Web page. Preliminary URL addresses are: 
http://www.uio.no/ ajaunsen/index.html

http://www.stsci.edu/ftp/stsci/library/grav_lens/grav_lens.html.

\section{Statistical model}

As in previous statistical works on optical samples of QSOs (Surdej et al. 1993a, Kochanek 1993, Jaunsen et al. 1995), we have used a simple statistical model, where the lens galaxy is described by means of a Singular Isothermal Sphere (SIS). We recall hereafter the main formalism, but refer the reader to previous publications for further details.

The optical depth or probability for lensing, in an Einstein-de-Sitter Universe is, for a SIS lens model, given by:

$$
\tau=\frac{1}{30} F D_{s}^{3}\left(z_{s}\right)
$$

where $\mathrm{F}$ is the lens effectiveness parameter $\left(F \propto n_{0} \sigma_{*}^{4}\right.$ with $n_{0}$ being the local spatial density of galaxies (E/S0-type), $\sigma_{*}$ is the one-dimensional velocity dispersion of the galaxy, and $D_{s}$ is the proper distance in a flat universe).

An important effect that must be taken into consideration when computing the frequency of lensing in the universe is the well known magnification bias. The bias stems from the fact that QSOs dimmer than the target QSO are amplified to brighter luminosities due to the effects of lensing. The expected frequency of lensing is therefore higher than that directly estimated from the general optical depth. The magnification bias can be estimated for each QSO from its magnitude $m_{i}$ by the following expression:

$$
\operatorname{Bias}\left(m_{i}, M_{M i n}\left(r_{s}\right), \infty\right)=\int_{M_{M i n}\left(r_{s}\right)}^{\infty} \frac{8}{M^{3}} \frac{n_{q}\left(m_{i}+2.5 \log M(r)\right)}{n_{q}\left(m_{i}\right)} d M
$$

The total integrated bias is computed within the range of the survey search angular radius, $r_{s}$. For each radius, a corresponding minimum magnification $M_{M i n}\left(r_{s}\right)$ (or maximum magnitude difference) is found using the survey angular selection function (ASF). The ASF is determined for each instrumental configuration (i.e. each survey sample) and is determined through simulations (cf. Surdej et al. 1993a).

Finally, the probability for observing a lensed QSO, taking into account the general optical depth, magnification bias and detectability, is expressed by

$$
p_{i}^{S F}\left(m_{i}, z_{i}\right)=\frac{S_{c a t} F}{30} D_{s}^{3}\left(z_{i}\right) \int_{0}^{r_{s} / 2} p_{c}\left(b_{c r}\right) \operatorname{Bias}\left(m_{i}, M\left(2 b_{c r}\right), M_{2}\right) d b_{c r}
$$


$S_{c a t}=0.7$ is the correction factor for incompleteness of the sample, due to the fact that QSO surveys loose about $30 \%$ of GL-systems (Kochanek 1991). $p_{c}\left(b_{c r}\right)$ represents the probability for a SIS lens galaxy to make a lens with angular separation $2 b_{c r}$, and $M_{2}$ is a finite (large) constant to prevent any divergence in equation (2), when the slope of the QSO number counts is changed.

\section{Maximum likelihood analysis}

\subsection{INTRODUCTION}

The maximum likelihood (ML) technique is a powerful tool to set constraints on parameters of the statistical model, using all the observed properties of the sample. So far, we have taken into account the frequency of lenses in the optical sample and the observed angular separation between their images (described by the configuration probability $p_{\text {Conf }}$; see Kochanek 1993, Jaunsen et al. 1995). We have also included a configuration probability, $p_{C o n f, R a d}$, for the $N_{L, \text { Radio }}$ radio lenses whose ASF is known. $p_{C o n f, R a d}$ is the probability of the observed angular separation between the lensed radio images, normalized by all the detectable angular separations. So, using a Poissonian statistics for the occurrence of lenses, we can write the logarithmic likelihood function to be maximized as:

$$
\ell=\ln L=-\sum_{i=1}^{N_{U}} p_{i}+\sum_{j=1}^{N_{L}} \ln p_{j}+\sum_{j=1}^{N_{L}} \ln p_{\text {Conf }, \mathrm{Obs}, j}+\sum_{j=1}^{N_{L, \text { Radio }}} \ln p_{\text {Conf }, \text { Rad }, j}
$$

where $N_{L}$ and $N_{U}$ are the number of lensed and unlensed objects in the sample.

\subsection{INPUT DATA}

The input data in the model are listed hereafter. These are also the default or reference values when the parameters are kept constant in the ML analysis.

$-n_{E l l}=0.005 \mathrm{~h}^{3} \mathrm{Mpc}^{-3}$ (Fukugita \& Turner 1991)

- $n_{\text {Spiral }}=0.015 \mathrm{~h}^{3} \mathrm{Mpc}^{-3}$ (Fukugita \& Turner 1991)

- $\sigma_{E l l}^{*}=220 \mathrm{~km} / \mathrm{s}$ (Fukugita \& Turner 1991)

- $\sigma_{\text {Spiral }}^{*}=143 \mathrm{~km} / \mathrm{s}$ (Fukugita \& Turner 1991)

$-\alpha=-1.1$ exponent of the Schechter luminosity function

- Tully-Fisher exponent $\gamma_{E l l}=4$

- Tully-Fisher exponent $\gamma_{\text {Spiral }}=2.6$ 

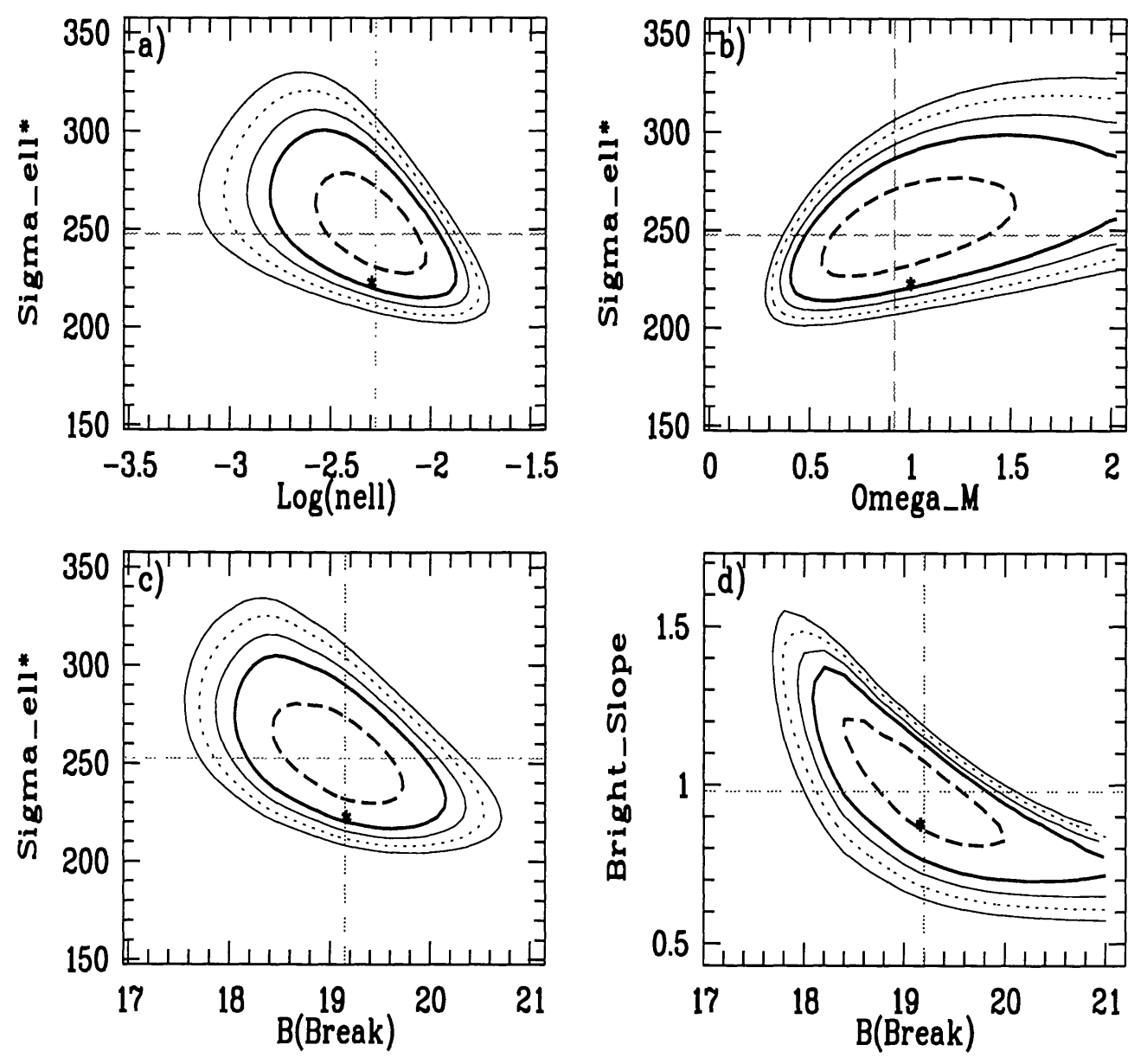

Figure 2. Maximum likelihood results: a) $\left(n_{E l l}, \sigma_{E l l}^{*}\right)$ plane; b) $\left(\Omega_{M}, \sigma_{E l l}^{*}\right)$ plane (flat universe); c) (B(Break), $\left.\sigma_{E l l}^{*}\right)$ plane; d) (B(Break), Bright end slope) plane.

- Magnitude break, bright end slope and faint end slopes of the differential number counts of QSOs (B band): 19.15, $0.86 \& 0.28$ resp. (Hartwick \& Schade 1990)

- Cosmological Model: Einstein - de- Sitter $\left(\Omega_{M}=1, \lambda_{o}=0\right)$

- Angular radius for the search of secondary images around the QSOs: $r_{s}=3^{\prime \prime}$

- Observed sample: 1528 observations of 1178 distinct HLQs

- 6 optical lenses (with $\Delta \theta \leq 3^{\prime \prime}$ ) (see Section 2)

- 10 radio lenses (with $\Delta \theta \leq 3^{\prime \prime}$ ): MG0414+053, B1422+231, B1938+666, B0218+356, MG1131+045, MG1654+134, MG1549+304 (Kochanek 1993, Refsdal \& Surdej 1994), and F10214+4724 (Lehár \& Broadhurst 1995), Q1600+434 (Jackson et al. 1995), Q1608+656 (Myers 1995). 


\subsection{RESULTS}

Figure 2 displays the likelihood contours in 4 different parameter spaces. The most inner solid (dashed) heavy contours are such that their projections onto the one dimensional axes give the $90 \%$ (68\%) confidence level intervals for the corresponding parameters. The dashed cross indicates the ML estimate of the parameters, and the star shows their reference values (given in Section 4.2).

We infer the following $90 \%$ confidence intervals for the parameters:

- Figure 2a): $n_{E l l}^{*} \in[0.0017,0.012] h_{100}^{3} \mathrm{Mpc}^{-3}$, and $\sigma_{E l l}^{*} \in[215,300]$ $\mathrm{km} / \mathrm{s}$. The resulting estimate of the efficiency parameter $\mathrm{F}$ for the ellipticals is 0.037 , and is well inside the $[0.012-0.070]$ range at the $90 \%$ confidence.

- Figure 2b): The upper limit on the cosmological constant in a flat universe is 0.55 at the $90 \%$ confidence.

- Figure 2c) \& d) The parameters B(Break) and the bright end slope exponent of the double power law for the surface density of QSOs belong respectively to $[18,20]$ and $[0.7,1.4]$ (at the $90 \%$ confidence level).

\section{Conclusions}

We have reviewed in this paper the present status of large optical samples of HLQs. The total merged sample contains 1178 distinct objects among which there are 6 lenses and a few more very promising GL candidates with small angular separations (e.g. the doubly imaged QSO J03.13), which are being found using precise PSF subtraction techniques. The ML analysis shows that the lensing statistics are fully compatible with independent estimates of galactic parameters (without any correction factor to account for dark matter in the halo), and with the standard number counts of QSOs (double power law). The upper value on the cosmological constant is 0.55 at the $90 \%$ confidence level. A better knowledge of our sample will still improve these constraints. For that, we need i) to identify all the lenses present in the sample; ii) to know the redshift of all the lensing galaxies (with the Keck and VLT telescopes).

\section{References}

Claeskens, J.F., Surdej, J., \& Remy, M., 1995, A\&A, submitted

Crampton, D., McClure, R., \& Fletcher, J., 1992, ApJ, 392, 23

Fukugita, M., \& Turner, E., 1991, MNRAS, 253, 99

Hartwick, F., \& Schade, D., 1990, ARA\&A, 28, 437

Jackson, N., De Bruyn, A.G., Myers, S., Bremer, M.N., et al., 1995, MNRAS, 274, L25

Jaunsen, A.O., Jablonski, M., Pettersen, B.R., \& Stabell, R., 1995, A\&A, in press 
Kochanek, C.S., 1991, ApJ, 379, 517

Kochanek, C.S., 1993, ApJ, 419, 12

Lehár, J., \& Broadhurst, T., 1995, in these proceedings

Magain, P., Surdej, J., Swings, J.-P., Borgeest, U., Kayser, R., Kühr, H., Refsdal, S., \& Remy, M., 1988, Nature, 334, 325

Magain, P., Surdej, J., Vanderriest, C., et al., 1992, A\&A, 253, L13 (Erratum: 1993, A\&A, 272, 383)

Maoz, D., Bahcall, J. N., Schneider, D. P., Bahcall, N. A., Djorgovski, S., et al., 1993, ApJ, 409, 28

Myers, S.T., 1995, in these proceedings

Refsdal, S. \& Surdej, J., 1994, Report on Progress in Phys, 128, 295

Surdej, J., Magain, P., Swings, J.-P., Borgeest, U., Courvoisier, T.J.-L., Kayser, R., Kellermann, K.I., Kühr, H., \& Refsdal, S., 1987, Nature, 329, 695

Surdej, J., Magain, P., Swings, J.P., et al., 1988, in Large Scale Structures: Observations and Instrumentations, eds. C. Balkowski \& S. Gordon

Surdej, J., Claeskens, J.-F., Crampton, D., Filippenko, A.V., Hutsemékers, D., et al., 1993a, AJ, 105, 2064

Surdej, J., Remy, M., Smette, A., et al., 1993b, in Gravitational lenses in the Universe, eds. J. Surdej, D. Fraipont-Caro, E. Gosset, S. Refsdal \& M. Remy, (Liège: Université de Liège) 153

Surdej, J., et al., 1995, in these proceedings

Turner, E., Ostriker, J., \& Gott, J., 1984, ApJ, 284, 1

Weymann, R., Latham, D., Angel, J., Green, R., Liebert, J., Turnshek, D., \& Tyson, J., 1980, Nature, 285, 641

Wisotski, L., Koehler, T., Kayser, R., \& Reimers, D., 1993, A\&A, 278, L15

Yee, H., Filippenko, A., \& Tang, D., 1993, AJ, 105, 7 